\title{
Trace formula for dielectric cavities. III. TE modes
}

\author{
E. Bogomolny \\ Université Paris Sud, CNRS, LPTMS, UMR 8656, Orsay F-91405, France
}

R. Dubertrand

School of Mathematics, University Walk, University of Bristol, Bristol BS8 1TW, United Kingdom

(Received 15 June 2012; published 10 August 2012)

\begin{abstract}
The construction of the semiclassical trace formula for resonances with transverse electric polarization for two-dimensional dielectric cavities is discussed. Special attention is given to the derivation of the two first terms of Weyl's series for the average number of such resonances. The formulas obtained agree well with numerical calculations for dielectric cavities of different shapes.
\end{abstract}

DOI: 10.1103/PhysRevE.86.026202

PACS number(s): 05.45.Mt, 42.55.Sa, 03.65.Sq

\section{INTRODUCTION}

Open dielectric cavities have attracted great interest in recent years due to their numerous and potentially important applications $[1,2]$. From a theoretical point of view, the crucial difference between dielectric cavities and the much more investigated case of closed quantum billiards [3-5] is that in the latter the spectrum is discrete but in the former it is continuous. Indeed, the main subject of investigations in open systems is not the true spectrum but the spectrum of resonances defined as poles of the scattering $S$ matrix (see, e.g., [6,7]).

The wavelength of the electromagnetic field is usually much smaller than any characteristic cavity size (except its height), and semiclassical techniques are useful and adequate for a theoretical approach to such objects. It is well known that the trace formulas are a very powerful tool in the semiclassical description of closed systems; see, e.g., [3-5]. Therefore, the generalization of trace formulas to different open systems, in particular to dielectric cavities, is of importance.

The trace formula for resonances with transverse magnetic (TM) polarization in two-dimensional (2D) dielectric cavities has been developed in Ref. [8] and shown to agree well with experiments and numerical calculations $[9,10]$. This paper is devoted to the construction of the trace formula for 2D dielectric cavities but for transverse electric (TE) polarization. Due to different boundary conditions the case of TE modes differs in many aspects from that of TM modes. In particular, special treatment is required for the resonances related to Brewster's angle [11] at which the Fresnel reflection coefficient vanishes.

Our main result is the asymptotic formula in the semiclassical (or short wavelength) regime for the average number of TE resonances for a $2 \mathrm{D}$ dielectric cavity with refraction index $n$, area $\mathcal{A}$, and perimeter $\mathcal{L}$,

$$
\bar{N}_{\mathrm{TE}}(k)=\frac{\mathcal{A} n^{2} k^{2}}{4 \pi}+r_{\mathrm{TE}}(n) \frac{\mathcal{L} k}{4 \pi}+o(k) .
$$

Here $\bar{N}_{\mathrm{TE}}(k)$ is the mean number of resonances (defined below) whose real part is less than $k$, the coefficient $r_{\mathrm{TE}}$ is given by the expression

$$
\begin{aligned}
r_{\mathrm{TE}}(n)= & 1+\frac{1}{\pi} \int_{-\infty}^{\infty} \tilde{R}_{\mathrm{TE}}(t)\left(\frac{n^{2}}{n^{2}+t^{2}}-\frac{1}{1+t^{2}}\right) d t \\
& +\frac{2 n}{\sqrt{n^{2}+1}}
\end{aligned}
$$

and $\tilde{R}_{\mathrm{TE}}$ is the Fresnel reflection coefficient for scattering on a straight dielectric interface at imaginary momentum

$$
\tilde{R}_{\mathrm{TE}}(t)=\frac{\sqrt{n^{2}+t^{2}}-n^{2} \sqrt{1+t^{2}}}{\sqrt{n^{2}+t^{2}}+n^{2} \sqrt{1+t^{2}}} .
$$

The plan of the paper is the following. In Sec. II the main equations describing the TE modes are recalled. In Sec. III the circular cavity is briefly reviewed: an exact quantization condition is derived, which allows a direct semiclassical treatment. In Sec. IV the first two Weyl terms for the resonance counting function are derived. It is important to notice that, for TE modes, one can have total transmission of a ray when the incidence angle is equal to Brewster's angle. This leads to a special set of resonances, which are counted separately in Sec. V. Section VI is devoted to a brief derivation of the oscillating part of the resonance density. In Sec. VII our obtained formulas are shown to agree well with numerical computation for cavities of different shapes. In the Appendix another method of deriving the Weyl series for TE polarization based on Krein's spectral shift formula is presented.

\section{GENERALITIES}

To describe a dielectric cavity correctly one should solve the three-dimensional Maxwell equations. In many applications the transverse height of a cavity, say along the $z$ axis, is much smaller than any other cavity dimensions. In this situation the three-dimensional problem in a reasonable approximation can be reduced to two 2D scalar problems (for each polarization of the field) following the so-called effective index approximation; see, e.g., $[12,13]$ for more details.

In the simplest setting, when one ignores the dependence of the effective index on frequency, such a 2D approximation consists in using the Maxwell equations for an infinite cylinder. It is well known [11] that in this geometry the Maxwell equations are reduced to two scalar Helmholtz equations inside and outside the cavity:

$$
\begin{aligned}
\left(\Delta+n^{2} k^{2}\right) \Psi(\vec{x}) & =0, \quad \vec{x} \in \mathcal{D}, \\
\left(\Delta+k^{2}\right) \Psi(\vec{x}) & =0, \quad \vec{x} \notin \mathcal{D},
\end{aligned}
$$

where $n$ is the refractive index of the cavity, $\mathcal{D}$ indicates the interior of the dielectric cavity, and $\Psi=E_{z}$ for the TM polarization and $\Psi=B_{z}$ for the TE polarization. 
The Helmholtz equations (4) have to be completed by the boundary conditions. The field $\Psi(\vec{x})$ is continuous across the cavity boundary and its normal derivatives along both sides of the boundary are related for two polarizations as [11]

$$
\left.\frac{\partial \Psi}{\partial v}\right|_{\text {from inside }}=\left\{\begin{array}{lll}
\left.\frac{\partial \Psi}{\partial v}\right|_{\text {from outside }} & \text { for } & \text { TM } \\
\left.n^{2} \frac{\partial \Psi}{\partial v}\right|_{\text {from outside }} & \text { for } & \text { TE. }
\end{array}\right.
$$

Open cavities have no true discrete spectrum. Instead, we are interested in the discrete resonance spectrum, which is defined as the (complex) poles of the $S$ matrix for the scattering on a cavity (see, e.g., [7]). It is well known that the positions of the resonances can be determined directly by solution of the problem (4) and (5) by imposing the outgoing boundary conditions at infinity:

$$
\Psi(\vec{x}) \propto e^{i k|\vec{x}|}, \quad|\vec{x}| \rightarrow \infty .
$$

The set (4)-(6) admit complex eigenvalues $k$ with $\operatorname{Im} k<0$, which are the resonances of the dielectric cavity and are the main object of this paper. Our goal is to count such resonances for the TE polarization in the semiclassical regime. This will provide us with the analog of Weyl's law derived for closed systems; see, e.g., [14].

\section{CIRCULAR CAVITY}

The circular dielectric cavity is the only finite 2D cavity which permits an analytical solution. Let $R$ be the radius of such a cavity. Writing $\Psi(\vec{x})=A J_{m}(n k r) e^{i m \phi}$ inside the cavity and $\Psi(\vec{x})=B H_{m}^{(1)}(k r) e^{i m \phi}$ outside the cavity, it is plain to see that, in order to fulfill the boundary conditions, it is necessary that $k$ is determined from the equation $s_{m}(x)=0$ with $x=k R$ and

$$
s_{m}(x)=x\left[\frac{1}{n} J_{m}^{\prime}(n x) H_{m}^{(1)}(x)-J_{m}(n x) H_{m}^{(1) \prime}(x)\right],
$$

where $J_{m}(x)\left[H_{m}^{(1)}(x)\right]$ denotes the Bessel function (the Hankel function of the first kind). Here and below the prime indicates the derivative with respect to the argument. The factor $x$ in Eq. (7) is introduced for further convenience. Equation (7) can also be seen as the quantization condition for the TE modes of the circular dielectric cavity.

Using $J_{m}(x)=\left[H_{m}^{(1)}(x)+H_{m}^{(2)}\right] / 2$ the equation $s_{m}(x)=0$ can be rewritten in the form

$$
R_{m}(x) E_{m}(x)=1,
$$

where

$$
E_{m}(x)=\frac{H_{m}^{(1)}}{H_{m}^{(2)}}(n x)
$$

and

$$
R_{m}(x)=\frac{\frac{H_{m}^{(1) \prime}}{H_{m}^{(1)}}(n x)-n \frac{H_{m}^{(1) \prime}}{H_{m}^{(1)}}(x)}{-\frac{H_{m}^{(2) \prime}}{H_{m}^{(2)}}(n x)+n \frac{H_{m}^{(1) \prime}}{H_{m}^{(1)}}(x)} .
$$

In the semiclassical limit, $x \rightarrow \infty$, the asymptotic formula for the Hankel function [15] $(0 \leqslant m \leqslant x)$ gives

$$
H_{m}^{(1)}(x) \simeq \frac{\sqrt{2 / \pi}}{\left(x^{2}-m^{2}\right)^{1 / 4}} e^{i \Phi_{m}(x)}\left[1+O\left(x^{-1}\right)\right],
$$

where

$$
\Phi_{m}(x)=\sqrt{x^{2}-m^{2}}-m \arccos \left(\frac{m}{x}\right)-\frac{\pi}{4} .
$$

In this way one obtains

$$
E_{m} \underset{x \rightarrow \infty}{\longrightarrow} e^{2 i \Phi_{m}(n x)}
$$

and

$$
R_{m}(x) \underset{x \rightarrow \infty}{\longrightarrow} R_{\mathrm{TE}}\left(\frac{m}{x}\right),
$$

where $R_{\mathrm{TE}}$ is the standard TE Fresnel coefficient for the scattering on an infinite dielectric interface,

$$
R_{\mathrm{TE}}(t)=\frac{\sqrt{n^{2}-t^{2}}-n^{2} \sqrt{1-t^{2}}}{\sqrt{n^{2}-t^{2}}+n^{2} \sqrt{1-t^{2}}} .
$$

It may be worth recalling that from the usual WKB approach the angular quantum number $m$ is related to the classical incidence angle $\theta$ by $m=n x_{1} \sin \theta$. The above formulas mean that in the semiclassical limit, Eq. (8) takes the form

$$
R_{\mathrm{TE}}\left(\frac{m}{x}\right) e^{2 i \Phi_{m}(n x)}=1
$$

or

$$
\Phi_{m}(n x)=\pi p+\frac{i}{2} \ln R_{\mathrm{TE}}\left(\frac{m}{x}\right)
$$

with integer $p=0,1,2, \ldots$.

In fact, this equation is valid in the semiclassical limit for closed and open circular cavities with other boundary conditions as well. The only difference is that, instead of the Fresnel reflection coefficient $R_{\mathrm{TE}}$, it is necessary to use the reflection coefficient for the problem under consideration. For example, for closed billiards, $n=1$ and for Neumann (Dirichlet) boundary conditions $R_{m}(x)$ in Eq. (14) equals 1 $(-1)$. For an open dielectric circular cavity with the TM polarization $R_{m}(x) \rightarrow R_{\mathrm{TM}}(m / x)$, where $R_{\mathrm{TM}}$ is the usual Fresnel reflection coefficient for the TM modes [11],

$$
R_{\mathrm{TM}}(t)=\frac{\sqrt{n^{2}-t^{2}}-\sqrt{1-t^{2}}}{\sqrt{n^{2}-t^{2}}+\sqrt{1-t^{2}}} .
$$

\section{WEYL TERMS}

Semiclassical formulas like Eq. (16) are convenient to obtain the average number of eigenvalues and resonances for closed and open systems with different boundary conditions. Let us consider first the simplest case of a closed billiard with Neumann boundary conditions for which $R_{m}(x)=1$. In the semiclassical regime the eigenvalues for this model are determined from Eq. (17) which reads

$$
\Phi_{m}(x)=\pi p,
$$

where $\Phi_{m}(x)$ is defined in Eq. (12) and $p=0,1, \ldots$ is an integer. Therefore, for fixed $m$, the number of eigenvalues less than $x$ is $\left[N_{m}(x)\right]$ where [ $]$ stands for the integer part and

$$
N_{m}(x)=\frac{1}{\pi} \Phi_{m}(x)+1 .
$$


1 is added as the integer $p$ in Eq. (19) starts with 0 but $\left[N_{m}(x)\right]$ has to begin with 1 .

Summing over all $m$ leads to the total number of eigenvalues less than $x$, usually called the counting function. This sum is finite as the asymptotics (11) is valid when $|m| \leqslant x$. Finally

$$
N(x)=\sum_{m=-[x]}^{[x]}\left[N_{m}(x)\right] .
$$

The averaged number of levels is determined from the equation

$$
\bar{N}(x)=\sum_{m=-x}^{x}\left[N_{m}(x)-\frac{1}{2}\right] .
$$

With the needed precision one can substitute the summation over $m$ by an integral and, consequently, the averaged number of eigenvalues for a circular billiard with Neumann boundary conditions can be approximated as follows:

$\bar{N}(x)=2 \int_{0}^{x}\left\{\frac{1}{\pi}\left[\sqrt{x^{2}-m^{2}}-m \arccos \left(\frac{m}{x}\right)\right]+\frac{1}{4}\right\} d m$.

Using the formula

$$
\int_{0}^{1}\left[\sqrt{1-t^{2}}-t \arccos (t)\right] d t=\frac{\pi}{8}
$$

one gets

$$
\bar{N}(x)=\frac{1}{4} x^{2}+\frac{1}{2} x+O(1) .
$$

As for the circle the area is $\mathcal{A}=\pi R^{2}$ and the perimeter is $\mathcal{L}=2 \pi R$, these results can be rewritten in the standard form Ref. [14]

$$
\bar{N}(k)=\frac{\mathcal{A} k^{2}}{4 \pi}+r \frac{\mathcal{L} k}{4 \pi}+O(1)
$$

with $r=1$. For Dirichlet boundary conditions similar arguments show that $1 / 4$ in Eq. (23) is substituted by $-1 / 4$ and $r=-1$, as it should be Ref. [14].
For open cavities Eq. (16) gives complex solutions (resonances) $k=k_{1}+i k_{2}$ with negative imaginary part, $k_{2}<0$. In the semiclassical limit for all investigated cases one has $\left|k_{2}\right| \ll k_{1}$. Separating the imaginary and real parts in Eq. (17) and using that

$$
\frac{\partial}{\partial x} \Phi_{m}(x)=\sqrt{1-\frac{m^{2}}{x^{2}}}
$$

one gets that in the first order in $k_{2}$ the real part of the resonance position, $k_{1}$ (or $x_{1}=k_{1} R$ ), is determined from the following real equation similar to Eq. (17):

$$
\Phi_{m}\left(n x_{1}\right)+\delta\left(m / x_{1}\right)=\pi p, \quad p \text { integer, }
$$

where $2 \delta(t)$ is the argument of the reflection coefficient,

$$
R(t)=|R(t)| e^{2 i \delta(t)} .
$$

In the same approximation the imaginary part of the resonance position, $k_{2}$, is

$$
k_{2} R=\frac{\ln \left|R\left(x_{1}\right)\right|}{2 \sqrt{n^{2}-m^{2} / x_{1}^{2}}} .
$$

This semiclassical approximation is quite good even for not too large $m$ as indicated in Fig. 1.

The above arguments demonstrate that the total number of resonances can be calculated from the real equation (28). As in Eq. (22) one concludes that the mean number of resonances with real part $x_{1}$ less than $x$ is given by the expression

$$
\bar{N}(x)=\sum_{m=-n x}^{n x}\left[N_{m}(x)-\frac{1}{2}\right]
$$

with

$$
N_{m}(x)=\frac{1}{\pi} \Phi_{m}(n x)+\frac{1}{\pi} \delta\left(\frac{m}{x}\right)+1 .
$$

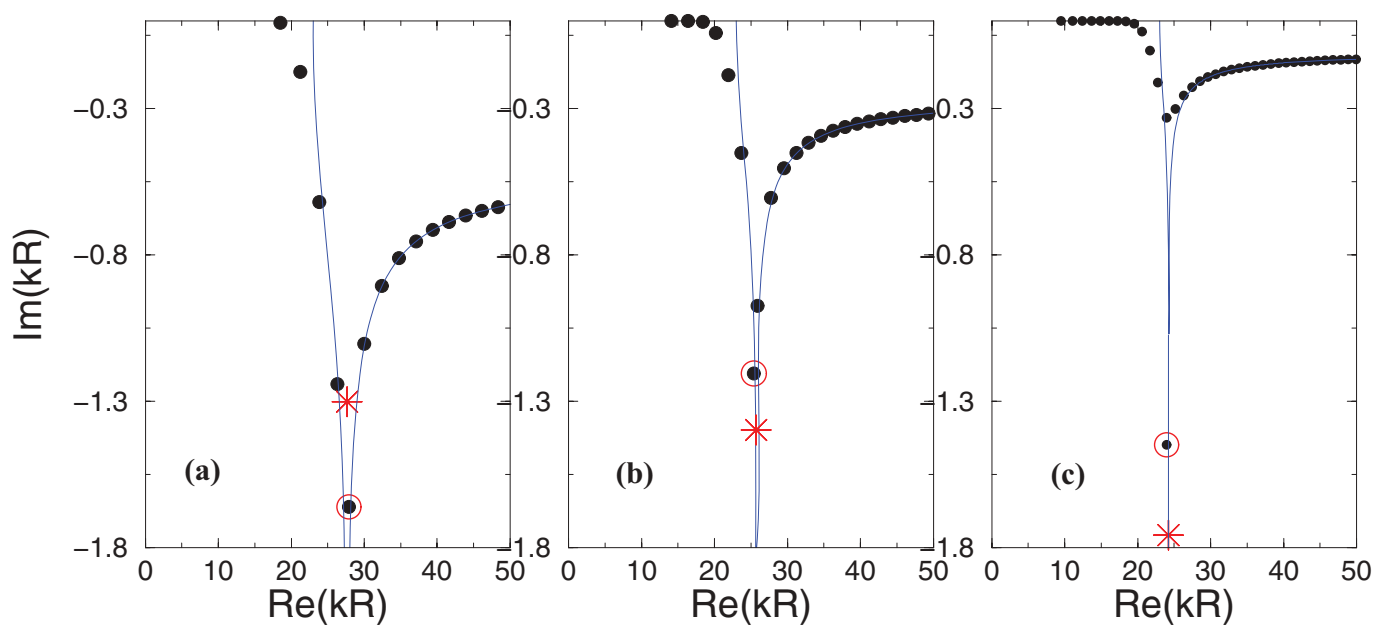

FIG. 1. (Color online) The black circles are the exact positions of the resonances with $m=23$ and $n=1.5$ (a), $n=2$ (b), and $n=3$ (c). The blue full lines indicate the approximation (30). The additional levels are encircled by the red circles. The red stars show the approximate formula (48). 
Consider first the case of TM modes. The reflection coefficient in this case is given by Eq. (18) and one has

$$
\delta_{\mathrm{TM}}(t)= \begin{cases}-\arctan \left(\frac{\sqrt{t^{2}-1}}{\sqrt{n^{2}-t^{2}}}\right), & 1 \leqslant t \leqslant n, \\ 0, & 0 \leqslant t \leqslant 1 .\end{cases}
$$

Therefore

$$
\begin{aligned}
\bar{N}(x)= & 2 \int_{0}^{n x} \frac{1}{\pi}\left[\sqrt{n^{2} x^{2}-m^{2}}-m \arccos \left(\frac{m}{n x}\right)\right] d m \\
& +2 \int_{0}^{n x}\left[\frac{1}{\pi} \delta_{\mathrm{TM}}\left(\frac{m}{x}\right)+\frac{1}{4}\right] d m=\frac{n^{2}}{4} x^{2}+\frac{n}{2} x \\
& -\frac{2 x}{\pi} \int_{1}^{n} \arctan \left(\frac{\sqrt{t^{2}-1}}{\sqrt{n^{2}-t^{2}}}\right) d t
\end{aligned}
$$

By integration by parts and contour deformation it is easy to check that

$$
\begin{aligned}
& 2 \int_{1}^{n} \arctan \left(\frac{\sqrt{t^{2}-1}}{\sqrt{n^{2}-t^{2}}}\right) d t \\
& \quad=\frac{\pi}{2}(n-1)-\int_{0}^{\infty} \tilde{R}_{\mathrm{TM}}(t)\left(\frac{n^{2}}{n^{2}+t^{2}}-\frac{1}{1+t^{2}}\right) d t,
\end{aligned}
$$

where $\tilde{R}_{\mathrm{TM}}(t)$ is the same as Eq. (18) but for the purely imaginary argument

$$
\tilde{R}_{\mathrm{TM}}(t) \equiv R_{\mathrm{TM}}(i t)=\frac{\sqrt{n^{2}+t^{2}}-\sqrt{1+t^{2}}}{\sqrt{n^{2}+t^{2}}+\sqrt{1+t^{2}}}
$$

Finally, these considerations lead to an expression similar to Eq. (26):

$$
\bar{N}_{\mathrm{TM}}(k)=\frac{\mathcal{A} n^{2} k^{2}}{4 \pi}+r_{\mathrm{TM}} \frac{\mathcal{L} k}{4 \pi}+o(k),
$$

where

$$
r_{\mathrm{TM}}(n)=1+\frac{1}{\pi} \int_{-\infty}^{\infty} \tilde{R}_{\mathrm{TM}}(t)\left(\frac{n^{2}}{n^{2}+t^{2}}-\frac{1}{1+t^{2}}\right) d t,
$$

which agrees with the result in [8] obtained by a different method.

Consider now TE modes. In this case the reflection coefficient is given by Eq. (15) and its argument is

$$
\delta_{\mathrm{TE}}(t)= \begin{cases}-\arctan \left(\frac{n^{2} \sqrt{t^{2}-1}}{\sqrt{n^{2}-t^{2}}}\right), & 1 \leqslant t \leqslant n, \\ 0, & t^{*} \leqslant t \leqslant 1, \\ -\frac{\pi}{2}, & 0 \leqslant t \leqslant t^{*},\end{cases}
$$

where $t^{*}$ corresponds to the zero of the TE reflection coefficient (Brewster's angle), $R_{\mathrm{TE}}\left(t^{*}\right)=0$,

$$
t^{*}=\frac{n}{\sqrt{n^{2}+1}} \text {. }
$$

Using these values we get

$$
\bar{N}_{\mathrm{TE}}(k)=\frac{\mathcal{A} n^{2} k^{2}}{4 \pi}+r_{\mathrm{TE}} \frac{\mathcal{L} k}{4 \pi}+o(k),
$$

where $r_{\mathrm{TE}}$ is given by the following expression:

$$
r_{\mathrm{TE}}(n)=-\frac{4}{\pi} \int_{1}^{n} \arctan \left(\frac{n^{2} \sqrt{t^{2}-1}}{\sqrt{n^{2}-t^{2}}}\right) d t+n-2 \int_{0}^{t^{*}} d t
$$

Similarly to Eq. (35) one can prove that

$$
\begin{aligned}
& 2 \int_{1}^{n} \arctan \left(n^{2} \frac{\sqrt{t^{2}-1}}{\sqrt{n^{2}-t^{2}}}\right) d t \\
& \quad=\frac{\pi}{2}(n-1)-\int_{0}^{\infty} \tilde{R}_{\mathrm{TE}}(t)\left(\frac{n^{2}}{n^{2}+t^{2}}-\frac{1}{1+t^{2}}\right) d t
\end{aligned}
$$

where, as above, $\tilde{R}_{\mathrm{TE}}(t)$ is the TE reflection coefficient (15) analytically continued to imaginary $t$,

$$
\tilde{R}_{\mathrm{TE}}(t) \equiv R_{\mathrm{TE}}(i t)=\frac{\sqrt{n^{2}+t^{2}}-n^{2} \sqrt{1+t^{2}}}{\sqrt{n^{2}+t^{2}}+n^{2} \sqrt{1+t^{2}}}
$$

Combining all terms together we obtain that

$$
\begin{aligned}
r_{\mathrm{TE}}(n)= & 1+\frac{1}{\pi} \int_{-\infty}^{\infty} \tilde{R}_{\mathrm{TE}}(t)\left(\frac{n^{2}}{n^{2}+t^{2}}-\frac{1}{1+t^{2}}\right) d t \\
& -\frac{2 n}{\sqrt{n^{2}+1}} .
\end{aligned}
$$

The first two terms are the same as for TM modes (38) but with the TE reflection coefficient. The last term is the new one related to the change of the sign of the TE reflection coefficient.

Higher-order terms in Weyl's expansions (37) and (41) are not yet calculated so we prefer to use a conservative estimate of them as $o(k)$ although all numerical checks suggest that for smooth boundary cavities it is $O(1)$.

\section{ADDITIONAL RESONANCES}

Formula (45) is the correct description for resonances for which the real part of the eigenmomentum $k$ corresponds to a nonzero reflection coefficient (i.e., $m / x_{1} \neq t^{*}$ ). This is due to the fact that when the reflection coefficient is zero its phase is not defined. For TE modes there is a special branch of resonances for which semiclassically the real part does obey $m / x_{1}=t^{*}$. The existence of such additional resonances was first discussed in a different context in Ref. [16].

The approximate positions of these resonances can be calculated as follows. Assume that the resonances have a large imaginary part. As $H_{m}^{(2)}(x-i \tau)$ tends to zero when $\tau \rightarrow \infty$ one can approximate Eq. (7) by

$$
\tilde{s}_{m}(x)=0, \quad \tilde{s}_{m}(x)=\frac{H_{m}^{(1) \prime}}{H_{m}^{(1)}}(n x)-n \frac{H_{m}^{(1) \prime}}{H_{m}^{(1)}}(x) .
$$

From Eq. (11) it follows that

$$
\frac{H_{m}^{(1) \prime}}{H_{m}^{(1)}}(x) \underset{x \rightarrow \infty}{\longrightarrow} i \sqrt{1-\frac{m^{2}}{x^{2}}}-\frac{x}{2\left(x^{2}-m^{2}\right)} .
$$

Using this expression one concludes that the solution of the equation $\tilde{s}_{m}\left(\tilde{x}_{m}\right)=0$ has the form

$$
\tilde{x}_{m} \approx \frac{\sqrt{n^{2}+1}}{n} m-i \frac{\left(n^{2}+1\right)^{3 / 2}}{2 n^{2}} .
$$

This approximation is better for large $n$ when the imaginary part is large but it gives reasonable results even for $n$ of the order of 1 . In practice one may use (48) as the initial value for any root search algorithm (cf. Fig. 1).

From Eq. (48) it follows that the ratio $m / \tilde{x}_{m}$ tends to $t^{*}$ defined in Eq. (40) so these resonances are not taken explicitly into account in Eq. (45). Their number can be estimated 
as follows. The discussed resonances correspond to waves propagating along the boundary whose direction forms an angle with the normal exactly equal to Brewster's angle,

$$
\sin \theta_{\mathrm{B}}=\frac{n}{\sqrt{n^{2}+1}} .
$$

If the length of the boundary is $\mathcal{L}$, the possible values for the momenta of such states in the semiclassical limit are

$$
k_{m} \sin \theta_{\mathrm{B}}=\frac{2 \pi}{\mathcal{L}} m
$$

with integer $m=0, \pm 1, \pm 2, \ldots$. Therefore, the number of additional resonances related to Brewster's angle is

$$
N_{\text {add }}(k) \approx \frac{\mathcal{L} k}{\pi} \frac{n}{\sqrt{n^{2}+1}} .
$$

Comparing it with Eq. (45) we conclude that for a general cavity the second term in the Weyl expansion for the averaged number of resonances for TE polarization is the following:

$$
\begin{aligned}
r_{\mathrm{TE}}= & 1+\frac{1}{\pi} \int_{-\infty}^{\infty} \tilde{R}_{\mathrm{TE}}(t)\left(\frac{n^{2}}{n^{2}+t^{2}}-\frac{1}{1+t^{2}}\right) d t \\
& \pm \frac{2 n}{\sqrt{n^{2}+1}},
\end{aligned}
$$

where the plus sign is used when the above additional resonances are taken into account and the minus sign corresponds to the case when these resonances are ignored.

For small values of $n$ the additional resonances are mixed with other resonances and their separation seems artificial. For large $n$ the additional branch of resonances is well separated from the main body of resonances and one can decide not to take them into account. In this case, the minus sign has to be used in Eq. (52) (see below Sec. VII).

When the cavity remains invariant under a group of symmetry it is often convenient to split resonances according to their symmetry representations. For reflection symmetries it is equivalent to considering a smaller cavity where along parts of the boundary one has to impose either Dirichlet or Neumann boundary conditions. In this case the total boundary contribution to the average counting function $\bar{N}(k)$ is given by the general formula

$$
\frac{1}{4 \pi}\left[n\left(L_{\mathrm{N}}-L_{\mathrm{D}}\right)+r_{\mathrm{TE}}(n) L_{0}\right] k .
$$

Here $L_{\mathrm{N}}$ and $L_{\mathrm{D}}$ are the lengths of the boundary parts with respectively Neumann and Dirichlet boundary conditions and $L_{0}$ is the length of the true dielectric interface. It is this formula which will be used in Sec. VII for dielectric cavities in the shapes of a square and a stadium.

\section{OSCILLATING PART OF THE TRACE FORMULA}

The quantization conditions (7) or (8) permit us also to obtain the resonance trace formula for a circular dielectric cavity. Let $k_{j}=k_{1 j}-i k_{2 j}$ be resonance eigenmomenta. Define the density of resonances as follows:

$$
d(k)=-\frac{1}{\pi} \operatorname{Im} \sum_{j} \frac{1}{k-k_{j}}=\frac{1}{\pi} \sum_{j} \frac{k_{2 j}}{\left(k-k_{1 j}\right)^{2}+k_{2 j}^{2}} .
$$

In general, if $x_{j}$ are the zeros of a certain function $F(x)$ which has no other singularities then the density of these zeros (54) formally is given by the expression

$$
d(x)=-\frac{1}{\pi} \operatorname{Im} \frac{F^{\prime}(x)}{F(x)} .
$$

In the semiclassical limit $k \rightarrow \infty$ it is sufficient to consider the semiclassical formula (16), i.e., $F(x)=\prod_{m} F_{m}(x)$ and

$$
F_{m}(x)=1-R_{\mathrm{TE}}\left(\frac{m}{x}\right) e^{2 i \Phi_{m}(n x)} .
$$

A more careful discussion is performed in the Appendix. In this manner one gets

$$
\begin{aligned}
d^{(\mathrm{osc})}(k) & =\frac{2 R}{\pi} \sum_{m=-\infty}^{\infty} \operatorname{Re} \sqrt{n^{2}-\frac{m^{2}}{x^{2}}} \frac{R_{m} e^{2 i \Phi_{m}(n x)}}{1-R_{m} e^{2 i \Phi_{m}(n x)}} \\
& =\frac{R}{\pi} \sum_{m=-\infty}^{\infty} \sqrt{n^{2}-\frac{m^{2}}{x^{2}}} \sum_{r=1}^{\infty} R_{m}^{r} e^{2 r i \Phi_{m}(n x)}+\text { c.c. }
\end{aligned}
$$

Here $R_{m}=R_{\mathrm{TE}}(m / x)$ is the Fresnel reflection coefficient for TE polarization (15).

The further steps are as usual; see, e.g., [8]. Using the Poisson summation formula

$$
\sum_{m=-\infty}^{\infty} f(m)=\sum_{M=-\infty}^{\infty} \int_{m=-\infty}^{\infty} e^{2 \pi i M m} f(m) d m
$$

the expression (57) becomes

$$
\begin{aligned}
d^{(\mathrm{osc})}(k)= & \frac{R}{\pi} \sum_{M=-\infty}^{\infty} \int_{m=-\infty}^{\infty} d m \sqrt{n^{2}-\frac{m^{2}}{x^{2}}} \sum_{r=1}^{\infty} R_{m}^{r} e^{i S_{M, r}(m)} \\
& + \text { c.c. }
\end{aligned}
$$

where the action is

$$
S_{M, r}(m)=2 \pi M m+2 r \Phi_{m}(n x) .
$$

When $k \rightarrow \infty$ the dominant contribution to the integral is due to saddle point solutions $m_{\mathrm{sp}}$ determined from the equation $\partial S_{M, r}(m) / \partial m=0$. It is plain that

$$
m_{\mathrm{sp}}=n x \cos \left(\theta_{M, r}\right)
$$

with $\theta_{M, r}=\pi M / r$. This saddle point corresponds geometrically to a periodic orbit of the circle in the shape of regular polygon with $r$ vertices going around the center $M<r$ times. Expanding the action $S_{M, r}(m)$ around the saddle point (61) one gets

$$
S_{M, r}\left(m_{\mathrm{sp}}+\delta m\right) \approx n k l_{p}-\frac{\pi}{2} r+\frac{r}{n x \sin \theta_{M, r}}(\delta m)^{2} .
$$

Here $l_{p}=2 r R \sin \theta_{M, r}$ is the classical length of the periodic orbit determined by $M$ and $r$.

In the end one gets the trace formula for the resonances of the circular dielectric cavity in the form

$$
d^{(\mathrm{osc})}(k)=2 k \frac{n^{3 / 2}}{\pi} \sum_{M, r} \frac{\mathcal{A}_{p}}{\sqrt{2 \pi k l_{p}}} R_{p}^{r} e^{i\left[n k l_{p}-r \pi / 2+\pi / 4\right]}+\text { c.c. },
$$



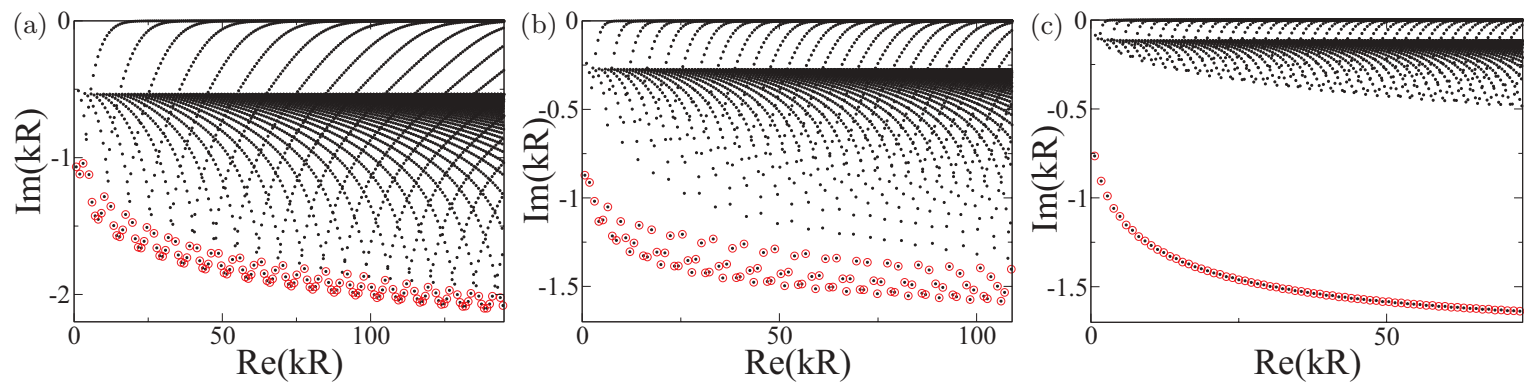

FIG. 2. (Color online) The black dots show the resonance spectra for TE modes of the circular dielectric cavity with $n=1.5$ (a), $n=2$ (b), and $n=3$ (c). The bottom red circles encircle the additional branch of resonances obtained by choosing initial conditions (48) and running a root searching routine to solve the equation $s_{m}(x)=0$ with $s_{m}(x)$ given by Eq. (7). The ranges along the $x$ axis are chosen such that every plot contains around 12000 resonances (counted with multiplicity).

where $\mathcal{A}_{p}=\pi R^{2} \sin ^{2} \theta_{M, r}$ is the area occupied by a given periodic orbit family, and $R_{p}=R_{\mathrm{TE}}\left(n \cos \theta_{M, r}\right)$ is the Fresnel reflection coefficient for the TE scattering with an angle equal to the reflection angle $\theta_{M, r}$ for the given periodic orbit.

Repeating the arguments presented in Eq. [8] we argue that in general the oscillating part of the resonance trace formula in the strong semiclassical limit has the form of a sum over all classical periodic orbits,

$$
d(E)=\sum_{p} d_{p}(E)+\text { c.c. },
$$

where the contribution of an individual orbit depends on the orbit considered: times

(a) For an isolated primitive periodic orbit $p$ repeated $r$

$$
d_{p}(E)=\frac{n l_{p}}{\pi\left|\operatorname{det}\left(M_{p}^{r}-1\right)\right|^{1 / 2}} R_{p}^{r} e^{i r n k l_{p}-i r \mu_{p} \pi / 2},
$$

where $l_{p}, M_{p}, \mu_{p}$, and $R_{p}$ are, respectively, the length, the monodromy matrix, the Maslov index, and the total TE Fresnel reflection coefficient for the chosen primitive periodic orbit.

(b) For a primitive periodic orbit family

$$
d_{p}(E)=\frac{n^{3 / 2} \mathcal{A}_{p}}{\pi \sqrt{2 \pi k l_{p}}}\left\langle R_{p}^{r}\right\rangle e^{i n k l_{p}-i r \mu_{p} \pi / 2}
$$

where $\mathcal{A}_{p}$ is the area covered by one periodic orbit family, and $\left\langle R_{p}^{r}\right\rangle$ is the mean value of the TE Fresnel reflection coefficient averaged over a periodic orbit family.
The only difference from the corresponding results derived in Ref. [8] is that the TE reflection coefficient is used instead of the TM coefficient.

\section{NUMERICAL VERIFICATION}

The numerical calculations of the resonance spectrum for the TE modes of the circular dielectric cavity are presented in Fig. 2. Notice that when the cavity refraction index $n$ increases the additional branch of the resonances (48) separates more and more from the main part of the spectrum.

In Fig. 3 we plot the difference between the function counting the numerically computed resonances with a real part less than $k$ (with radius $R=1$ ) and the best fit to it of the form [see Eq. (41)]

$$
N_{\text {fit }}(k)=\frac{n^{2}}{4}(k R)^{2}+a_{1}(k R)+a_{0},
$$

where $a_{1}$ and $a_{0}$ are fitting parameters.

For $n=1.5$ and $n=2$ we consider all resonances including the additional branch. For $n=3$ this branch is quite far from the other resonances [cf. Fig. 2(c)] and it is natural not to include it in the counting. The fitted values of the parameters for these three cases are the following:

$$
n=1.5, \quad a_{1}=1.246, \quad a_{0}=-0.66, \quad n=2, \quad a_{1}=1.122,
$$$$
a_{0}=-0.50, \quad n=3, \quad a_{1}=-1.189, \quad a_{0}=0.12 \text {. }
$$

The term $a_{1}$ has to be compared with the theoretical prediction which follows from Eq. (52) (used with a plus sign for $n=1.5$
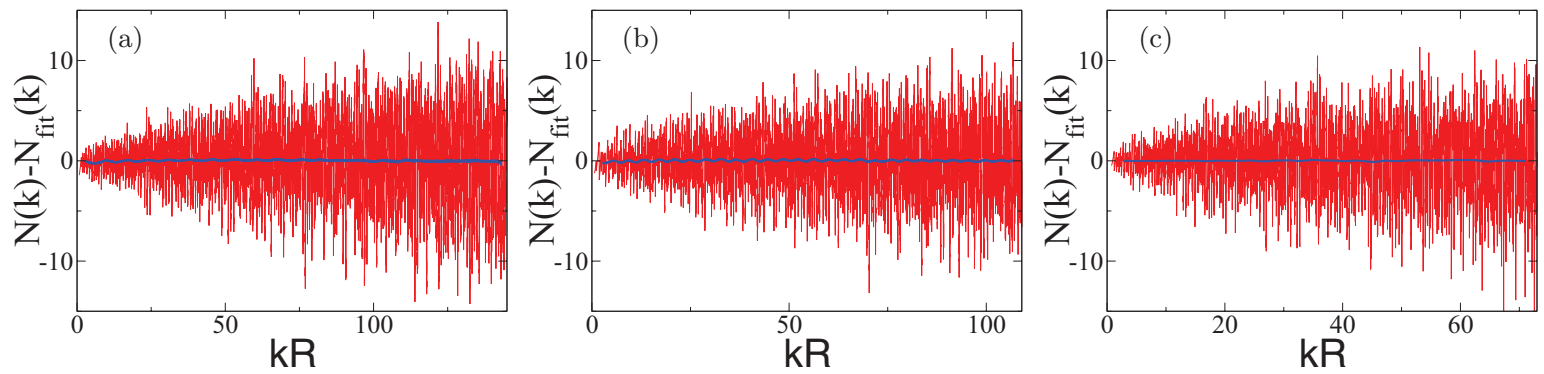

FIG. 3. (Color online) Difference between the exact number of resonances and the fit (67) for $n=1.5$ (a), $n=2$ (b), and $n=3$ (c). In the last case the additional resonances in Fig. 2(c) are not taken into account. The blue solid thick line in the center indicates the difference averaged over a large interval. 

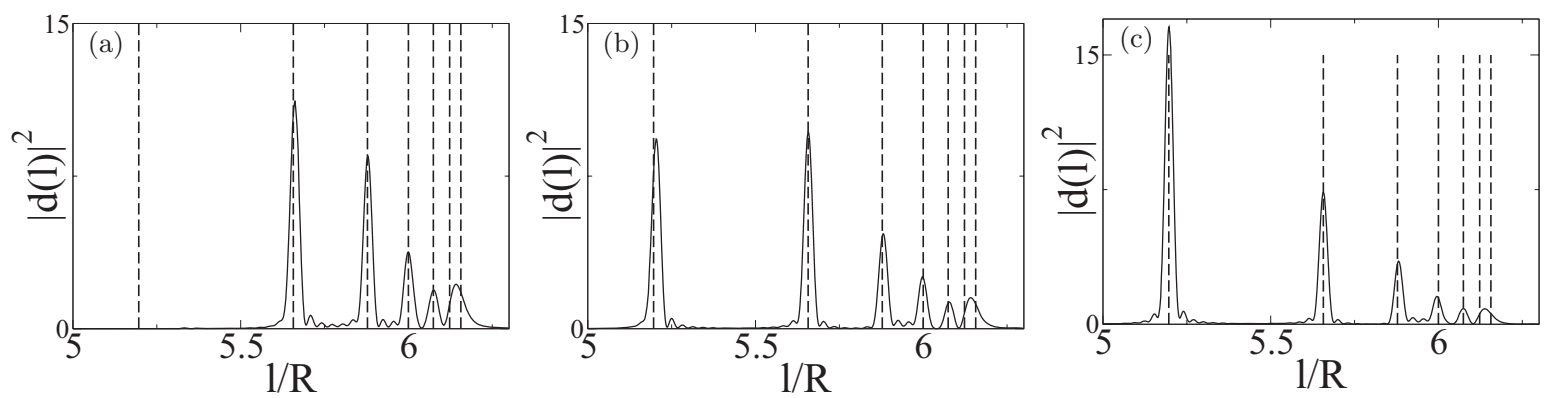

FIG. 4. Density of periodic orbit length (Fourier transform of the resonance density) for $n=1.5$ (a), $n=2$ (b), and $n=3$ (c). The vertical dashed lines indicate the length of the shortest periodic orbits of the circular cavity; from left to right, triangle, square, pentagon, hexagon, heptagon, and octagon.

and $n=2$, and with a minus sign for $n=3$ ):

$$
\begin{aligned}
& n=1.5, \quad a_{1}^{\text {th }}=1.247, \quad n=2, \quad a_{1}^{\text {th }}=1.124, \\
& n=3, \quad a_{1}^{\text {th }}=-1.190 .
\end{aligned}
$$

The agreement with our numerical calculations is very good.

In Fig. 4 the Fourier transform of the resonance density for the circular dielectric cavity with different values of the refractive index is displayed. As expected from the trace formula, this quantity has peaks at the lengths of classical periodic orbits of the circle. Notice especially that the triangular orbit is not confined for $n=1.5$. Hence the Fresnel reflection coefficient is small and induces damping, which can be clearly seen in Fig. 4(a). As the index grows it is also shown that the contribution of short-period orbits becomes closer and closer to that of the closed billiard.

Our results were also compared for two nonintegrable shapes. The following numerical results were obtained via the boundary integral method. Briefly speaking, this consists in discretizing the integral equations derived from Eqs. (4) and (5); see more details, e.g., in Ref. [17].

In Fig. 5(a) we present the spectrum of the TE resonances for the square cavity of side $a=1$ with $n=1.5$ and (-,-) symmetry along the diagonals. For such a cavity the fit function similar to Eq. (67) is

$$
N_{\mathrm{fit}}(k)=\frac{n^{2}}{16 \pi}(k a)^{2}+a_{1}(k a)+a_{0}
$$

and the best fit gives [see Fig. 5(b)]

$$
a_{1}=0.0304, \quad a_{0}=-5.22 .
$$

Due to the low value of the index the additional resonances are also taken into account: the + sign in Eq. (52) is chosen. The theoretical prediction for this symmetry class is obtained from Eq. (53) as $a_{1}^{\text {th }}=0.0297$, which agrees well with the numerical calculations.

Finally the same procedure was done for the dielectric stadium consisted of two half circles of radius $R$ connected by a rectangle with sides $2 \alpha R$ and $2 R$ where $\alpha$ is called the aspect ratio of the stadium. The calculations were restricted to the symmetry class such that the associated function vanishes along both symmetry axes of the stadium, which is again called the $(-,-)$ symmetry class. The resonance spectrum for $n=1.5$ is presented in Fig. 6(a).

The fit function is now

$$
N_{\mathrm{fit}}(k)=\frac{n^{2}}{4 \pi}\left(\alpha+\frac{\pi}{4}\right)(k R)^{2}+a_{1}(k R)+a_{0},
$$

where the aspect ratio $\alpha$ has been taken as 1 in the numerical calculations. The best fit gives [see Fig. 6(b)]

$$
a_{1}=0.150, \quad a_{0}=-5.24,
$$

which agrees well with the prediction for this symmetry class: $a_{1}^{\text {th }}=0.152$ [cf. Eq. (53)]. For the same reason as above the
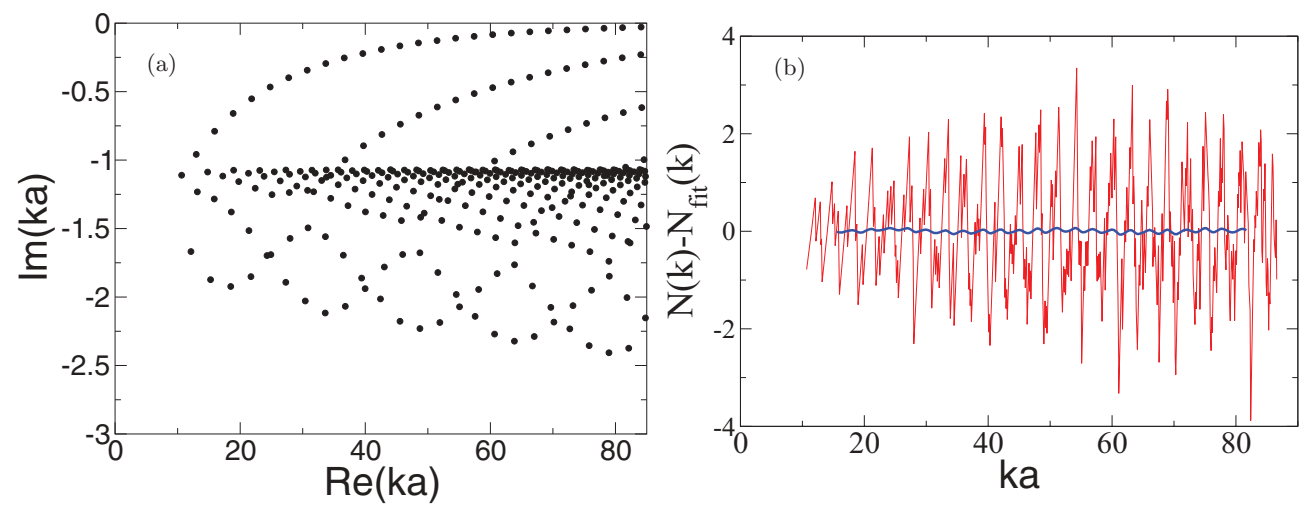

FIG. 5. (Color online) (a) Resonance spectrum for the dielectric square with $n=1.5$ for the $(-,-)$ symmetry class. (b) The difference between the total number of resonances and the best quadratic fit. The blue solid thick line in the center indicates the difference averaged over a large interval. 

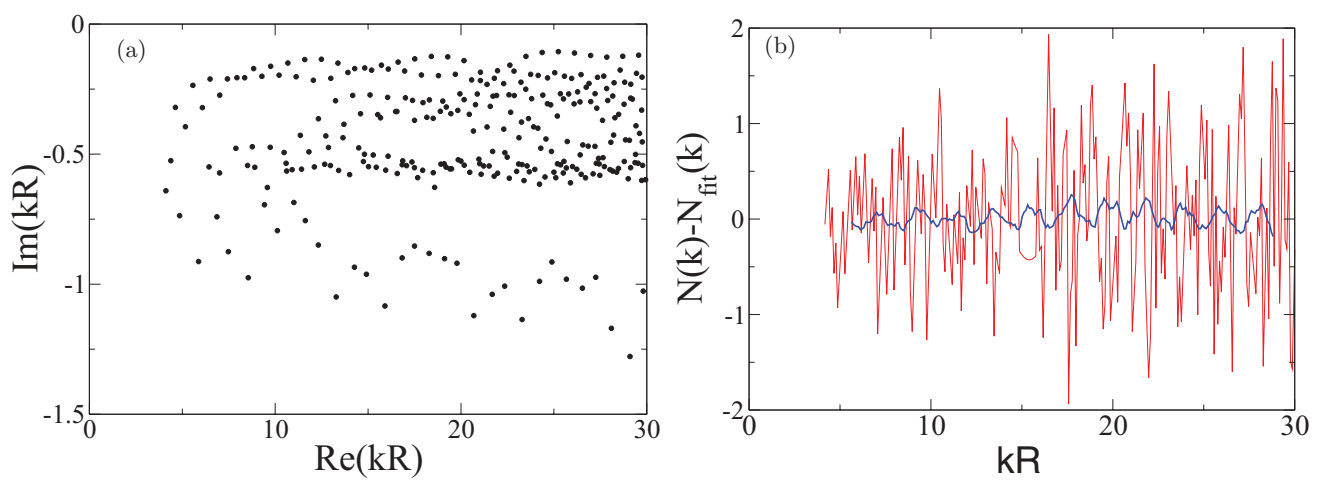

FIG. 6. (Color online) (a) Resonance spectrum for the dielectric stadium with $n=1.5$ for the $(-,-)$ symmetry class. (b) The difference between the total number of resonances and the best quadratic fit. The blue solid thick line in the center indicates the difference averaged over a large interval.

additional resonances have been taken into account and the + sign chosen in Eq. (52).

\section{SUMMARY}

Trace formulas are the main tool of the semiclassical description of multidimensional quantum problems. For closed systems the trace formulas relate two objects: the quantum density of discrete states and a sum over classical periodic orbits,

$$
\begin{aligned}
d(E) \equiv & \sum_{n} \delta\left(E-E_{n}\right) \approx \bar{d}(E)+\sum_{\text {periodic orbits }} A_{p} e^{i S_{p}(E) / \hbar} \\
& + \text { c.c. },
\end{aligned}
$$

where $S_{p}(E)$ is the classical action over a periodic orbit and $\bar{d}(E)$ is the mean density of eigenenergies, averaged over a small window around $E$. For $2 \mathrm{D}$ billiards with area $\mathcal{A}$ and perimeter $\mathcal{L}$ this averaged density of states is

$$
\bar{d}(E)=\frac{\mathcal{A}}{4 \pi}+r \frac{\mathcal{L}}{8 \pi \sqrt{E}}+o\left(E^{-1 / 2}\right),
$$

where $r=1$ for the Neumann boundary conditions and $r=$ -1 for the Dirichlet ones.

For open quantum models the true eigenenergy spectrum is continuous and the main object of interest is the discrete spectrum of resonances defined as the poles of the $S$ matrix in the complex plane: $E_{n}=e_{n}-i \Gamma_{n} / 2$ with $e_{n}$ and $\Gamma_{n}$ real. The real part of the resonance energy, $e_{n}$, gives the position of the resonance while its imaginary part, $\Gamma_{n}$, determines the resonance width.

The analog of the trace formula for open systems has a form similar to Eq. (74),

$$
\begin{gathered}
\frac{1}{\pi} \sum_{n} \frac{\Gamma_{n} / 2}{\left(E-e_{n}\right)^{2}+\Gamma_{n}^{2} / 4} \approx \bar{d}(E)+\sum_{\text {periodic orbits }} A_{p} e^{i S_{p}(E) / \hbar} \\
+ \text { c.c. . }
\end{gathered}
$$

In Ref. [8] such a formula has been obtained for a 2D dielectric cavity with transverse magnetic polarization of the field. Here we derive the trace formula for a $2 \mathrm{D}$ dielectric cavity but with boundary conditions corresponding to the transverse electric polarization of the electromagnetic field. As expected, the oscillating part of this trace formula is given by the usual periodic orbits weighted in the leading semiclassical order by the Fresnel coefficient corresponding to TE reflection on the cavity boundary (65) and (66).

Our main result is the expression for the average resonance density of a dielectric cavity with area $\mathcal{A}$, perimeter $\mathcal{L}$, and refraction index $n$,

$$
\bar{d}(E)=\frac{\mathcal{A} n^{2}}{4 \pi}+r_{\mathrm{TE}}(n) \frac{\mathcal{L}}{8 \pi \sqrt{E}}+o\left(E^{-1 / 2}\right),
$$

where

$$
\begin{aligned}
r_{\mathrm{TE}}(n)= & 1+\frac{1}{\pi} \int_{-\infty}^{\infty} \tilde{R}_{\mathrm{TE}}(t)\left(\frac{n^{2}}{n^{2}+t^{2}}-\frac{1}{1+t^{2}}\right) d t \\
& \pm \frac{2 n}{\sqrt{n^{2}+1}}
\end{aligned}
$$

and $\tilde{R}_{\mathrm{TE}}(t)$ is the Fresnel reflection coefficient for the TE polarization at imaginary momentum,

$$
\tilde{R}_{\mathrm{TE}}(t)=\frac{\sqrt{n^{2}+t^{2}}-n^{2} \sqrt{1+t^{2}}}{\sqrt{n^{2}+t^{2}}+n^{2} \sqrt{1+t^{2}}} .
$$

The plus-minus sign in front of the last term in Eq. (78) is connected with the existence for the TE modes of an additional series of resonances related to Brewster's angle. As these resonances have large imaginary parts, they may be included or not in the counting function. For small values of $n$ additional resonances are mixed with other resonances and their separation is artificial. In this case the plus sign has to be used. For large $n$ the branch of additional resonances is well separated from the body of resonances and it is natural to ignore them. This corresponds to the minus sign in Eq. (78).

The results of this paper together with Ref. [8] demonstrate that semiclassical trace formulas can be derived and applied for open dielectric cavities in close similarity with closed billiards. Further investigation of trace formulas for other physical open systems is of considerable interest.

\section{ACKNOWLEDGMENTS}

It is a pleasure to thank Martin Sieber for fruitful discussions and Stefan Bittner for providing numerical data for the dielectric circle with $n=3$. 


\section{APPENDIX: KREIN FORMULA APPROACH}

The purpose of this appendix is to present another derivation of the number of resonances in a circular dielectric cavity based on the Krein spectral shift formula [18]. This approach was also suggested in Ref. [19]. The true eigenenergy spectrum for an open system is continuous and, consequently, the density of states for open quantum systems is infinite. Nevertheless, the difference between the density of states with a cavity and the density of states without the cavity is finite and is given by the Krein formula

$$
d(E)-d_{0}(E)=\frac{1}{2 \pi i} \frac{\partial}{\partial E} \ln \operatorname{det} \mathbf{S}(E)
$$

where $\mathbf{S}(E)$ is the $S$ matrix for the scattering on the cavity.

This formula is general and can be used for any type of short-range potential. We apply it for scattering on a circular dielectric cavity. It is easy to check that the $S$ matrix for the scattering on a 2D circular cavity with TE boundary conditions (5) is diagonal in polar coordinates and

$$
S_{m}(x)=-\frac{\tilde{s}_{m}(x)}{s_{m}(x)},
$$

where $s_{m}(x)$ is given by Eq. (7) and $\tilde{s}_{m}(x)$ differs from $s_{m}(x)$ by the change of $H_{m}^{(1)}(x)$ to $H_{m}^{(2)}(x)$ :

$$
\tilde{s}_{m}(x)=x\left[\frac{1}{n} J_{m}^{\prime}(n x) H_{m}^{(2)}(x)-J_{m}(n x) H_{m}^{(2) \prime}(x)\right] .
$$

From properties of the Bessel functions [15] it is straightforward to show that

$$
\begin{aligned}
\frac{s_{m}^{\prime}}{s_{m}}(x)= & -\frac{\left(n^{2}-1\right)}{n^{2}} \\
& \times \frac{J_{m}(n x) H_{m}^{(1)}(x) m^{2} / x^{2}+n J_{m}^{\prime}(n x) H_{m}^{(1) \prime}(x)}{J_{m}^{\prime}(n x) H_{m}^{(1)}(x) / n-J_{m}(n x) H_{m}^{(1) \prime}(x)} .
\end{aligned}
$$

Using the equality $J_{m}(x)=\left[H_{m}^{(1)}(x)+H_{m}^{(2)}\right] / 2$, this expression can be rewritten in the form

$$
\frac{s_{m}^{\prime}}{s_{m}}(x)=-\frac{n^{2}-1}{n^{2}} \frac{A_{m}(x)+B_{m}(x) E_{m}(x)}{C_{m}(x)\left[1-R_{m}(x) E_{m}(x)\right]},
$$

where $E_{m}(x)$ and $R_{m}(x)$ are defined in Eqs. (9) and (10), respectively, and

$$
\begin{gathered}
A_{m}(x)=\frac{m^{2}}{x^{2}}+n \frac{H_{m}^{(2) \prime}}{H_{m}^{(2)}}(n x) \frac{H_{m}^{(1) \prime}}{H_{m}^{(1)}}(x), \\
B_{m}(x)=\frac{m^{2}}{x^{2}}+n \frac{H_{m}^{(1) \prime}}{H_{m}^{(1)}}(n x) \frac{H_{m}^{(1) \prime}}{H_{m}^{(1)}}(x), \\
C_{m}(x)=\frac{H_{m}^{(2) \prime}}{n H_{m}^{(2)}}(n x)-\frac{H_{m}^{(1) \prime}}{H_{m}^{(1)}}(x) .
\end{gathered}
$$

Expanding this expression into series of $E_{m}(x)$ gives

$$
\frac{s_{m}^{\prime}}{s_{m}}(x)=Q_{m}(x)+P_{m} \sum_{r=1}^{\infty} R_{m}^{r}(x) E_{m}^{r}(x),
$$

where

$$
Q_{m}(x)=-\frac{\left(n^{2}-1\right) A_{m}(x)}{n^{2} C_{m}(x)}
$$

and

$$
P_{m}(x)=-\frac{4 i\left(n^{2}-1\right)\left[\frac{m^{2}}{n^{2} x^{2}}+\left(\frac{H_{m}^{(1) \prime}}{H_{m}^{(1)}}(x)\right)^{2}\right]}{\pi n^{2} x H_{m}^{(1)}(n x) H_{m}^{(2)}(n x) C_{m}(x) D_{m}(x)} .
$$

with

$$
D_{m}(x)=\frac{H_{m}^{(1) \prime}}{n H_{m}^{(1)}}(n x)-\frac{H_{m}^{(1) \prime}}{H_{m}^{(1)}}(x) .
$$

In the semiclassical limit $x \rightarrow \infty$ the above formulas are simplified by using the asymptotic of the Hankel function (11):

$$
\frac{H_{m}^{(1,2) \prime}}{H_{m}^{(1,2)}}(x) \underset{x \rightarrow \infty}{\longrightarrow} \pm i \sqrt{1-\frac{m^{2}}{x^{2}}}-\frac{x}{2\left(x^{2}-m^{2}\right)}+O\left(x^{-2}\right) .
$$

Consider first the smooth term (A10). From the identity

$$
\begin{gathered}
\left(\frac{1}{n^{2}} \sqrt{n^{2}-t^{2}}+\sqrt{1-t^{2}}\right)\left(\sqrt{n^{2}-t^{2}}-\sqrt{1-t^{2}}\right) \\
=\frac{n^{2}-1}{n^{2}}\left(t^{2}+\sqrt{n^{2}-t^{2}} \sqrt{1-t^{2}}\right)
\end{gathered}
$$

it is straightforward to check that

$$
\begin{aligned}
Q_{m}(x) \underset{x \rightarrow \infty}{\longrightarrow} & -i\left[\sqrt{n^{2}-\frac{m^{2}}{x^{2}}}-\sqrt{1-\frac{m^{2}}{x^{2}}}\right] \\
& -\frac{x}{2} R_{\mathrm{TE}}\left(\frac{m}{x}\right)\left[\frac{n^{2}}{n^{2} x^{2}-m^{2}}-\frac{1}{x^{2}-m^{2}}\right],
\end{aligned}
$$

where $R_{\mathrm{TE}}(t)$ is the Fresnel reflection coefficient for the TE polarization given by Eq. (15).

The difference between the density of states with a cavity and the one without the cavity averaged over an energy interval such that periodic orbit terms are small can be calculated from $Q_{m}(x)$ :

$$
\langle d(E)\rangle-d_{0}(E)=-\frac{R}{2 \pi k} \sum_{m=-\infty}^{\infty} \operatorname{Im} Q_{m}(x) .
$$

Changing the summation over $m$ to integration and turning the integration contour in the second term in Eq. (A15) in the complex plane to avoid poles, $m \rightarrow-i t$, leads to

$$
\begin{aligned}
& \langle d(E)\rangle-d_{0}(E) \\
& =\frac{R}{2 \pi k}\left[\int_{-n x}^{n x} \sqrt{n^{2}-\frac{m^{2}}{x^{2}}} d m-\int_{-x}^{x} \sqrt{1-\frac{m^{2}}{x^{2}}} d m\right] \\
& \quad+\frac{R x}{4 \pi k} \int_{-\infty}^{\infty} d t R_{\mathrm{TE}}\left(-i \frac{t}{x}\right)\left[\frac{n^{2}}{n^{2} x^{2}+t^{2}}-\frac{1}{x^{2}+t^{2}}\right] .
\end{aligned}
$$

Rescaling integration variables, one gets

$$
\begin{aligned}
\langle d(E)\rangle-d_{0}(E)= & \frac{\mathcal{A}}{4 \pi}\left(n^{2}-1\right)+\frac{\mathcal{L}}{8 \pi^{2} k} \int_{-\infty}^{\infty} d t \tilde{R}_{\mathrm{TE}}(t) \\
& \times\left[\frac{n^{2}}{n^{2}+t^{2}}-\frac{1}{1+t^{2}}\right],
\end{aligned}
$$

where $\mathcal{A}=\pi R^{2}$ and $\mathcal{L}=2 \pi R$ are the area and the perimeter of a circular cavity. 
This formula differs from the averaged total number of resonances (41) and (45). This is the consequence of the fact discussed in Ref. [8] for the case of TM modes that the $S$ matrix for the scattering on a cavity has an additional phase (and additional zeros) connected with the outside scattering on the impenetrable cavity.

The form of this "additional" $S$ matrix may be argued as follows. It is known that when a wave from outside the cavity scatters on a cavity it reflects with a reflection coefficient which differs by its sign from the reflection coefficient from inside the cavity (this is a consequence of current conservation). For the TE polarization the Fresnel reflection coefficient for scattering from a medium with the refraction index 1 on another medium with the refraction index $n$ is $-R_{\mathrm{TE}}$ where $R_{\mathrm{TE}}$ is given by Eq. (15). In the semiclassical region accessible in outside scattering, $|t|<1$, the reflection coefficient $-R_{\mathrm{TE}}(t)$ is real and the "effective" reflection coefficient corresponding to the scattering on an impenetrable cavity equals the sign of $-R_{\mathrm{TE}}(t)$ [cf. (39)],

$$
R_{\mathrm{TE}}^{(\mathrm{eff})}= \begin{cases}-1, & t^{*} \leqslant|t| \leqslant 1, \\ 1, & 0 \leqslant|t| \leqslant t^{*},\end{cases}
$$

where $t^{*}=n / \sqrt{n^{2}+1}$.

The reflection coefficient equals $-1(+1)$, corresponding to scattering with Dirichlet (Neumann) conditions on the cavity boundary. For a circular cavity the $S$ matrices with Dirichlet and Neumann boundary conditions are well known (see, e.g., Ref. [20]):

$$
S_{m}^{(\mathrm{D})}(x)=-\frac{H_{m}^{(2)}}{H_{m}^{(1)}}(x), \quad S_{m}^{(\mathrm{N})}(x)=-\frac{H_{m}^{(2) \prime}}{H_{m}^{(1) \prime}}(x) .
$$

The additional $S$ matrix for the TE scattering is thus formally

$$
\begin{aligned}
\operatorname{det}\left[S_{0}^{(\mathrm{TE})}(x)\right]= & \prod_{m=-\infty}^{-\left(m^{*}+1\right)} S_{m}^{(\mathrm{D})}(x) \prod_{m=-m^{*}}^{m^{*}} S_{m}^{(\mathrm{N})}(x) \\
& \times \prod_{m=m^{*}+1}^{\infty} S_{m}^{(\mathrm{D})}(x),
\end{aligned}
$$

where $m^{*}=\left[x n / \sqrt{n^{2}+1}\right]$.
To find the total phase of this additional $S$ matrix one can proceed as follows. To the leading order in the semiclassical limit $x \rightarrow \infty$ the Dirichlet and Neumann $S$ matrices (A20) can be calculated from Eq. (11). It gives

$$
S_{m}^{(\mathrm{D})}(x) \approx-e^{-2 i \Phi_{m}(x)}, \quad S_{m}^{(\mathrm{N})}(x) \approx e^{-2 i \Phi_{m}(x)},
$$

where $\Phi_{m}(x)$ is given by Eq. (12). This means that $S_{m}^{(\mathrm{N})}$ differs from $S_{m}^{(\mathrm{D})}$ only by its sign, which is another manifestation of the opposite sign of the reflection coefficient (A19). Therefore one can rewrite expression (A21) as follows:

$$
S_{0}^{(\mathrm{TE})} \approx S^{(\mathrm{D})} \prod_{m=-m^{*}}^{m^{*}}(-1) \approx S^{(\mathrm{D})} e^{ \pm 2 i \pi x n / \sqrt{n^{2}+1}},
$$

where $S^{(\mathrm{D})}=\prod_{m=-\infty}^{\infty} S_{m}^{(\mathrm{D})}$ is the full $S$ matrix for scattering on a cavity with the Dirichlet boundary condition. The \pm sign in the exponent reflects the ambiguity of the phase, $-1=e^{ \pm i \pi}$.

The calculation of the mean density of states related to the $S^{(\mathrm{D})}$ matrix is straightforward (see, e.g., Ref. [20]):

$$
d_{\mathrm{D}}(E)-d_{0}(E)=-\frac{\mathcal{A}}{4 \pi}-\frac{\mathcal{L}}{8 \pi k},
$$

and finally from Eq. (A23) and the Krein formula (A1) one finds that the change of the density of states due to the additional $S$ matrix (A21) is

$$
\begin{gathered}
\bar{d}_{0}(E)-d_{0}(E)=d_{\mathrm{D}}(E)-d_{0}(E) \pm \frac{\mathcal{L}}{8 \pi k} \frac{2 n}{\sqrt{n^{2}+1}} \\
=-\frac{\mathcal{A}}{4 \pi}-\frac{\mathcal{L}}{8 \pi k}\left(1 \mp \frac{2 n}{\sqrt{n^{2}+1}}\right) .
\end{gathered}
$$

The total density of resonances is thus the difference between (A18) and (A25). In the end one gets Eqs. (41) and (45). The ambiguity in the phase of the additional $S$ matrix corresponds to the possibility of including resonances related to Brewster's angle in the Weyl formula or not, which has been discussed in Sec. V.
[1] Optical Microcavities, edited by K. Vahala (World Scientific, Singapore, 2004).

[2] A. B. Matsko, Practical Applications of Microresonators in Optics and Photonics (CRC Press, Boca Raton, FL, 2009).

[3] R. Balian and C. Bloch, Ann. Phys. (NY) 60, 401 (1970); 64, 271 (1971); 69, 76 (1972).

[4] M. Gutzwiller, Chaos in Classical and Quantum Mechanics (Springer-Verlag, Berlin, 1990).

[5] F. Haake, Quantum Signatures of Chaos (Springer-Verlag, Berlin, 2001).

[6] P. Lax and R. S. Phillips, Scattering Theory (Springer, New York, 1963).
[7] R. G. Newton, Scattering Theory of Waves and Particles (Springer-Verlag, New York, 1982).

[8] E. Bogomolny, R. Dubertrand, and C. Schmit, Phys. Rev. E 78, 056202 (2008).

[9] E. Bogomolny, N. Djellali, R. Dubertrand, I. Gozhyk, M. Lebental, C. Schmit, C. Ulysse, and J. Zyss, Phys. Rev. E 83, 036208 (2011).

[10] S. Bittner, E. Bogomolny, B. Dietz, M. Miski-Oglu, P. Oria Iriarte, A. Richter, and F. Schäfer, Phys. Rev. E 81, 066215 (2010); S. Bittner, E. Bogomolny, B. Dietz, M. Miski-Oglu, and A. Richter, ibid. 85, 026203 (2012); S. Bittner, B. Dietz, R. Dubertrand, J. Isensee, M. Miski-Oglu, and A. Richter, ibid. 85, 056203 (2012). 
[11] J. D. Jackson, Classical Electrodynamics (Wiley, New York, 1999).

[12] M. Lebental, N. Djellali, C. Arnaud, J.-S. Lauret, J. Zyss, R. Dubertrand, C. Schmit, and E. Bogomolny, Phys. Rev. A 76, 023830 (2007).

[13] R. Dubertrand, E. Bogomolny, N. Djellali, M. Lebental, and C. Schmit, Phys. Rev. A 77, 013804 (2008); C. Gmachl, F. Capasso, E. E. Narimanov, J. U. Nöckel, A. D. Stone, J. Faist, D. L. Sivco, and A. Y. Cho, Science 280, 1556 (1998); I. Braun, G. Ihlein, F. Laeri, J. U. Nöckel, G. Schulz-Ekloff, F. Schüth, U. Vietze, Ö. Weiß, D. Wöhrle, Appl. Phys. B: Lasers Opt. 70, 335 (2000); V. A. Podolsky, E. Narimanov, W. Fang, and H. Cao, Proc. Natl. Acad. Sci. USA 101, 10498 (2004).

[14] H. P. Baltes and E. R. Hilf, Spectra of Finite Systems (Bibliographisches Institut, Mannheim, 1976).
[15] A. Erdelyi, Higher Transcendental Functions (McGraw-Hill, New York, 1955), Vol. II

[16] C. P. Dettmann, G. V. Morozov, M. Sieber, and H. Waalkens, Europhys. Lett. 87, 34003 (2009).

[17] R. Dubertrand, Ph.D. thesis, University of Paris-Sud, 2008, http://tel.archives-ouvertes.fr/docs/00/34/33/67/PDF/ pdfthese.pdf (in French).

[18] M. G. Krein, Matem. Sbornik 33, 597 (1953); Dokl. Akad. Nauk SSSR 144, 268 (1962) [Sov. Math. Dokl. 3, 707 (1962)].

[19] J. U. Nöckel and R. K. Chang, in Cavity-Enhanced Spectroscopies, edited by R. D. Van Zee and J. P. Looney, Experimental Methods in the Physical Sciences (Academic Press, San Diego, 2002).

[20] U. Smilansky and I. Ussishkin, J. Phys. A 29, 2587 (1996). 